\title{
Design of Digital Fast Corrector Power Supply for TPS Storage Ring
}

\author{
Yuanchen Chien, Baosheng Wang, Yongseng Wong, Chenyao Liu and Kuobin Liu* \\ National Synchrotron Radiation Research Center, 101 Hsin-Ann Road Hsinchu Science Park Hsinchu 30076. Taiwan, R.O.C. \\ *Corresponding author
}

\begin{abstract}
TPS (Taiwan Photon Source) is a 3rd generation synchrotron light source, which is aimed to provide high brightness beam source for various novel scientific researches. Beginning from the start of 2016, to improve the beam orbit stability, fast orbit feedback system (FOFB) is installed and tested to eliminate undesired disturbances from various sources. The FOFB system consists of fast corrector magnets, power supplies, beam position data and orbit compensation algorithm to suppress the beam orbit instability. The preliminary study has shown FOFB system is effective in stabilizing the beam position This paper describes a new type full-digital corrector power supply to serve as the driving source to feed the fast corrector magnet in the FOFB system, where both wide bandwidth and fast transient response are needed. The new power supply employs the same power stage of the analog fast corrector power supply, the current output regulation is redesigned and implemented in NIsbRIO9606, which embeds a real-time processor and a userreconfigurable FPGA (Field Programmable Gate Array) on a single module from National Instruments. The simulation and prototype result indicates that the new power supply can deliver operational bandwidth at about $10 \mathrm{KHz}$ and faster transient behavior compared to the along version of the fast corrector power supply.
\end{abstract}

Keywords-component; synchrotron; fast corrector; wide bandwidth; power supply

\section{INTRODUCTION}

TPS is a $3 \mathrm{GeV}$ synchrotron facility with very low emittance and thus is capable of generating ultra-brightness beam source for advanced scientific studies in a variety of areas such as molecular, material, life and medicine related subjects [1]. TPS is a concentric ring, in which booster and storage ring are built and located in the same tunnel. The 50 $\mathrm{MeV}$ electron beam coming out of the linear accelerator is ramped up to $3 \mathrm{GeV}$ with $3 \mathrm{~Hz}$ repetition rate in the booster ring and then extracted to the storage ring which has a circumference of $518.4 \mathrm{~m}$. The lattice of the TPS storage ring is composed of 24 double-bend cells with 6-fold symmetry. TPS had started commissioning since 2014. It has successfully delivered over $500 \mathrm{~mA}$ at the end of 2015 and is scheduled to be ready for user operation in 2016.

For it is designed to have a very small beam sizes both in the vertical and horizontal plane and it is required that the beam position stability is in the order of tens of $\mu \mathrm{m}$. In order to the achieve this strict beam position requirement and keep the beam travel in the defined closed orbit range, fast orbit feedback system (FOFB) has to be utilized [2].
Starting from 2016, the FOFB system is installed, tested and proved to be effective in maintaining the beam trajectory to the reference closed orbit by eliminating perturbations coming from different sources like magnet strength errors, alignment errors and vibration caused by water pipes close to the magnets and other sources [3]. FOFB mainly consists of 3 major key components: Beam Position Monitor (BPM), orbit compensation computation unit and corrector power supply with specialized fast and slow setting control interface. In FOFB, total 170 BPMs and 96 fast correctors (Horizontal and Vertical Combined Type) installed in the storage ring lattice, therefore 192 fast corrector power supplies are needed to provide kick strength to correct the beam position deviation when necessary.

At the present time, bipolar four-quadrant power converter modules with centralized corrector power supply controller (CPSC) are used. There are total 8 power modules each with independent analog PID controller and one CPSC placed in the middle of a racket. The digital current commands are fed from the FOFB system to CPSC, where these commands are translated to analog ones by high-resolution DACs (Digital-toAnalog Converter). The power module is able to deliver $10 \mathrm{~A}$ with $-3 \mathrm{~dB}$ bandwidth up to $3 \mathrm{KHz}$.

The FOFB system modifies the fast correctors with 10 $\mathrm{KHz}$ update rate. The maximum corrected current value is set at $1 \%$ of the $10 \mathrm{~A}$ output, which is equivalent to maximum 0.1 A per 100 us $(10 \mathrm{KHz})$. This means that for a maximum $0.1 \mathrm{~A}$ step correction, the rise time and settling time of the fast corrector power supply should fall within 100 us period.

Even these power modules work with the FOFB system currently, common problems associated the analog PID controller still exist. First, it is not easy to realize highresolution DACs with ultra-low noise. Second, tuning the PID control parameters for all the 192 power supplies is difficult considering the capacitor and resistor value tolerance and component variation of the precision amplifiers used to constitute the analog PID controller. Moreover, the rise and settling time of the step response do not meet the FOFB specifications and the working bandwidth is expected to be wider to further extend FOFB's position error suppression ability to cope with higher frequency beam position perturbation.

This paper describes the design and implementation of a fast corrector power supply with digital control loop based on embedded microprocessor and FPGA [5-6]. With the digital 
architecture, the time and frequency domain response for 192 power modules is expected to be much more consistent, the PID parameters are easier to adjust and besides these, the working bandwidth is extended and the rise time is reduced with proper controller design and switching scheme strategy. A prototype was also built to verify if its performances meet the desired requests.

\section{PROTOPYE DESIGN}

The prototype design is based on the fast corrector power converter module with analog PI control. The corrector power module is developed and constructed by the NSRRC's power supply group of NSRRC and the Center for Measurement Standards of Industrial Technology Research Institute (CMS, ITRI) [4].

The $\pm 10 \mathrm{~A} / \pm 50 \mathrm{~V}$ corrector magnet power converter adopts H-bridge topology with $30 \mathrm{kHz}$ switching frequency. MOSFETs with reduced $R_{d s}$ (MOSFET on resistance) and precision shunt resistor with ultra-low temperature drift and fast frequency response are used to increase the working bandwidth. As a result, the achievable $-3 \mathrm{~dB}$ bandwidth is 3.4 $\mathrm{kHz}$ and rise time of the small signal step response ( $0.1 \mathrm{~A}$ step) is about 142 us. However, the time domain performance of the analog fast corrector power supply still not fully satisfies the needs for the FOFB system with $10 \mathrm{KHz}$ update rate, which requires the rise time small signal step response to fall with 100 us (10 KHz update rate). Hence, in order to speed up the step transient performance and further extend the working bandwidth for better orbit deviation elimination, the controller part of the present fast corrector power module is redesigned in the digital domain.

\section{A. Power Circuit Topology}

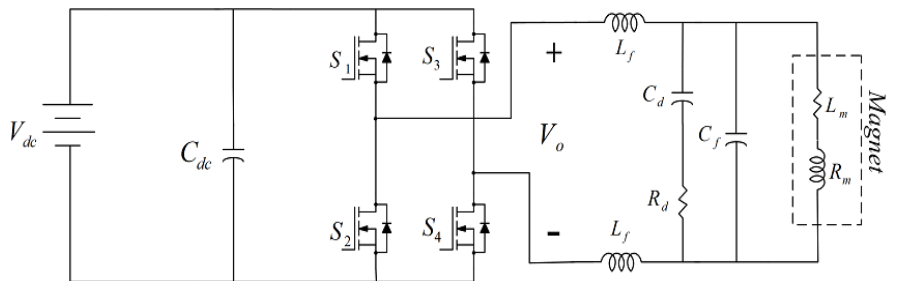

FIGURE I. POWER STAGE OF FAST CORRECTOR POWER SUPPLY

The equivalent circuit topology of the fast corrector power converter is shown in Figure I. The 48V DC comes from offthe-self $1.5 \mathrm{KW}$ AC-to-DC switching regulator and is connected to the DC bus terminals. The circuit is composed of a filter and energy storage capacitor, four MOSFET switches forming the H-bridge in two legs (S1/S3 and S2/S4) with embedded anti-parallel diode and damped output low pass filter.

The design parameters are listed in Table I.

TABLE I. DIGITAL FAST CORRECTOR POWER SUPPLY PARAMETERS

TPS Fast Corrector Output Specifications Maximum Current Output $\pm 10 \mathrm{~A}$

\begin{tabular}{|c|r|}
\hline Maximum Voltage Output & $\pm 50 \mathrm{~V}$ \\
\hline Low-pass Filter Parameters & \\
\hline$L_{f}$ & $100 \mu \mathrm{H}$ \\
\hline$C_{f}$ & $0.82 \mu \mathrm{F}$ \\
\hline$C_{d}$ & $33 \mu \mathrm{F}$ \\
\hline$R_{d}$ & $100 \mathrm{Ohm}$ \\
\hline$L_{m}$ & $2.92 \mathrm{mF}$ \\
\hline$R_{m}$ & $8.8 \mathrm{mOhm}$ \\
\hline
\end{tabular}

The $s$-domain transfer functions of the magnet load $\mathrm{G}_{L}(s)$ and the low pass filter $G_{L P F}(s)$ are expressed as follows:

$$
\mathrm{G}_{L}(s)=\frac{1}{R_{m}+L_{m} s}
$$

$$
G_{L P F}(s)=\frac{1+C_{d} \mathrm{R}_{d} s}{1+C_{d} \mathrm{R}_{d} s+\left(L_{f} C_{f}+L_{f} C_{d}\right) s^{2}+R_{d} C_{f} C_{d} L_{f} s^{3}}
$$

\section{B. Switching Methodology}

Complementary unipolar switching scheme is adopted for the Pulse-Width-Modulation (PWM) switching and the switching mechanism is shown in Figure II [7]. With this control technique, the MOSFETs of each leg are controlled independently of the other leg and the PWM control signals in the same leg are compliment to each other. The switching signals are such that S1, S2, S3 and S4 are generated by comparing the current command $V_{c}(t)$ and its negation $-V_{c}(t)$ to the reference triangular waveform $V_{t r i}(t)$. When $V_{c}(t)$ is greater than $V_{t r i}(t), \mathrm{S} 1$ is turned $\mathrm{ON}$, and when $-V_{c}(t)$ is greater than $V_{t r i}(t), \quad \mathrm{S} 3$ is $\mathrm{ON}$, while $\mathrm{S} 2$ and $\mathrm{S} 4$ are compliment to $\mathrm{S} 1$ and $\mathrm{S} 3$ respectively. The major benefit with this control scheme is that ripple frequency of the output current $I_{o}$ will be $100 \mathrm{KHz}$, which is twice that of the $50 \mathrm{KHz}$ PWM frequency and thus, the damped filter design is made easier while the filter component size can be reduced due to this ripple frequency doubling. The transfer function of the PWM scheme can be obtained as follows: Assume that during a switching period $T_{s}$, the control input $V_{c}(t)$ remains constant. 


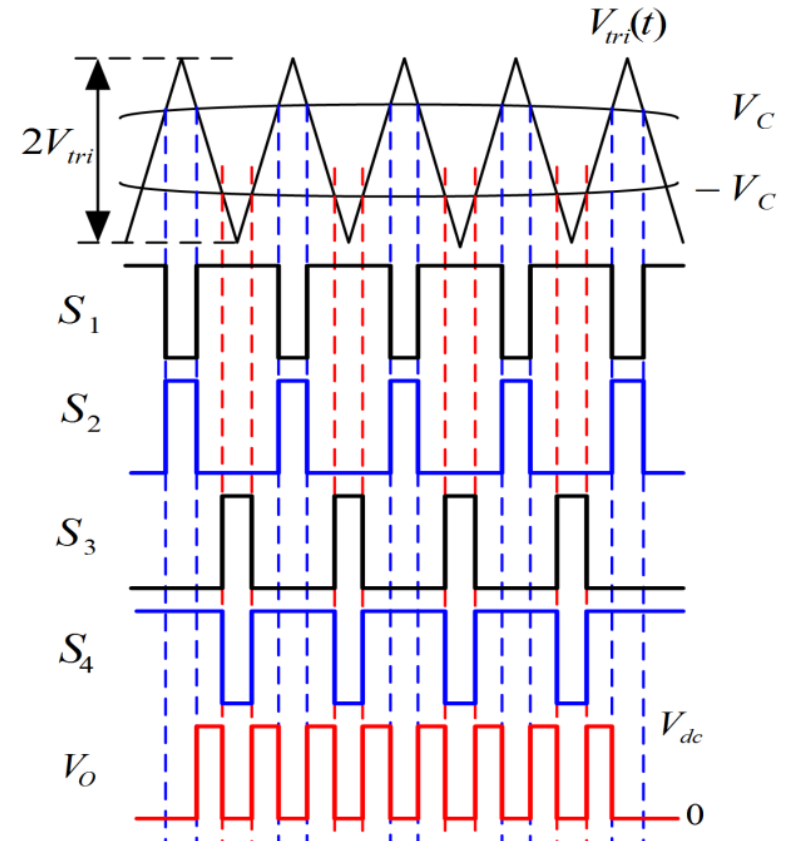

FIGURE II. UNIPOLAR SWITCHING DIAGRAM OF PWM

The relation of the on-duty ratio $d_{o n}$ and the PWM control input $V_{c}$ can be approximated and expressed as

$$
\begin{aligned}
& d_{o n}=\frac{1}{2}\left(1+\frac{V_{c}}{V_{t r i}}\right) \\
& d_{o f f}=1-d_{o n}
\end{aligned}
$$

where $V_{t r i}$ is half of the peak-to-peak amplitude of the PWM triangle waveform. And the averaged output voltage $V_{o}$ is given by

$$
\mathrm{V}_{\mathrm{o}}=\mathrm{d}_{\mathrm{on}} \mathrm{V}_{\mathrm{dc}}-\mathrm{d}_{\mathrm{off}} \mathrm{V}_{\mathrm{dc}}
$$

By replacing $d_{\text {off }}$ in equation (2) into equation (3), $V_{o}$ becomes

$$
\mathrm{V}_{\mathrm{o}}=\left(2 \mathrm{~d}_{\mathrm{on}}-1\right) \mathrm{V}_{\mathrm{dc}}
$$

Then substituting the $d_{o n}$ of equation (1) into equation (4),

$$
\mathrm{k}=\frac{\mathrm{v}_{\mathrm{o}}}{\mathrm{v}_{\mathrm{c}}}=\frac{\mathrm{v}_{\mathrm{dc}}}{\mathrm{v}_{\mathrm{tri}}}
$$

where $k$ is the derived transfer function of the complementary unipolar switching scheme.

\section{CONTROLLER DESIGN}

The overall closed-loop control system can be constructed by using derived transfer functions of the magnet load $G_{L}(s)$, the low pass filter $G_{L P F}(s)$ and the PWM converter gain $k$ obtained above. The block diagram of the closed loop system is depicted in Figure III.

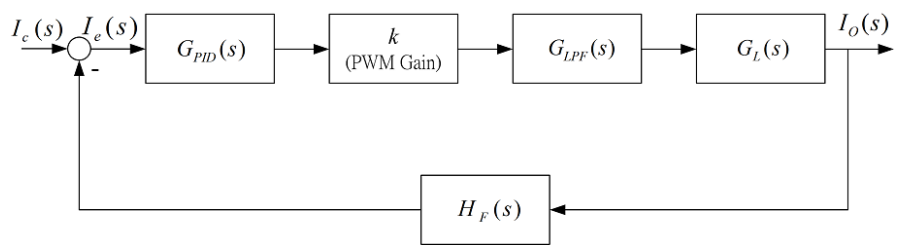

FIGURE III. BLOCK DIAGRAM OF THE CLOSED LOOP SYSTEM

The open loop transfer function $T_{\text {open }}(\mathrm{s})$ is expressed as

$$
T_{\text {open }}(\mathrm{s})=\frac{I_{e}(s)}{I_{o}(s)}=G_{P I D}(s) \cdot k \cdot G_{L P F}(s) \cdot G_{L}(s)
$$

Then the closed loop transfer function $T_{\text {closed }}(s)$ of the overall control system can be derived as

$$
\mathrm{T}_{\text {closed }}(s)=\frac{I_{c}(s)}{I_{o}(s)}=\frac{\mathrm{G}_{\mathrm{PID}}(\mathrm{s}) \cdot \mathrm{k} \cdot \mathrm{G}_{\mathrm{LPF}}(\mathrm{s}) \cdot \mathrm{G}_{\mathrm{L}}(\mathrm{s})}{1+\mathrm{G}_{\mathrm{PID}}(\mathrm{s}) \cdot \mathrm{k} \cdot \mathrm{G}_{\mathrm{LPF}}(\mathrm{s}) \cdot \mathrm{G}_{\mathrm{L}}(\mathrm{s})}
$$

where the $H_{F}(s)$ represents the feedback gain and is assumed as 1 .

The controller is designed using the Ziegler-Nichols method with the assistance of Simulink/Matlab toolbox. Figure IV shows the mathematical simulation model with Simulink. This is a mixed mode model simulation, where the transfer functions of each block is obtained as above in sdomain except the PID block, which is expressed in discrete zdomain. An AD converter (analog-to-digital converter) model, which is equivalent to a Zero-Order-Hold, is used to convert

\begin{tabular}{|c|c|}
\hline \multicolumn{2}{|c|}{$\begin{array}{c}\text { Fast Corrector Converter Controller Time Domain Design } \\
\text { Specifications }\end{array}$} \\
\hline Maximum step size & $0.1 \mathrm{~A}$ ( $1 \%$ of full scale $10 \mathrm{~A}$ output) \\
\hline Rise time & $<100$ us \\
\hline Overshoot & $5 \%$ of the Maximum step size \\
\hline Settling time & $<100$ us within $3 \%$ of Maximum step size \\
\hline
\end{tabular}
the analog feedback current to discrete data format with 50 $\mathrm{KHz}$ sampling frequency.

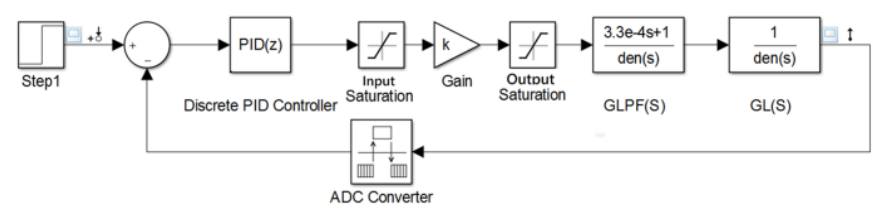

FIGURE IV. SIMULINK SIMULATION MODEL

The design goals to be achieved is listed in Table II.

TABLE II. TIME DOMAIN DESIGN SPICIFICATIONS

The $K_{P}$ (proportional), $K_{I}$ integral and $K_{D}$ derivative values of the discrete PID controller are tuned according to the specification using the automatic tuning tool in Simulink. The 
z-domain transfer function of the PID controller using Forward Euler representation can expressed as

$$
\begin{gathered}
G_{P I D}(z)=P+I * \frac{T_{s}}{2} \frac{1}{z-1}+D * \frac{T_{s}}{2} \frac{z-1}{z} \\
K_{P}=P \quad K_{I}=I * \frac{T_{s}}{2} \quad K_{D}=D * \frac{T_{s}}{2}
\end{gathered}
$$

where $T_{s}$ is $20 \mathrm{us,}$ which is the $50 \mathrm{KHz}$ sampling period. And the final tuned gain values are listed in Table III.

TABLE III. TUNED CONTROLLER GAIN OF PID CONTROLLER

\begin{tabular}{|c|c|c|}
\hline \multicolumn{3}{|c|}{ Digital PID Controller Parameter } \\
\hline$K_{P}$ & $K_{I}$ & $K_{D}$ \\
\hline 111 & 0.4727 & 0.000125 \\
\hline
\end{tabular}

The $0.1 \mathrm{~A}$ step response of the fast corrector magnet with the tuned PID controller values is shown in Figure V. The risetime is at 63.26 us, which is less than the 100 us constraint and other specifications are also met with the tuned values.

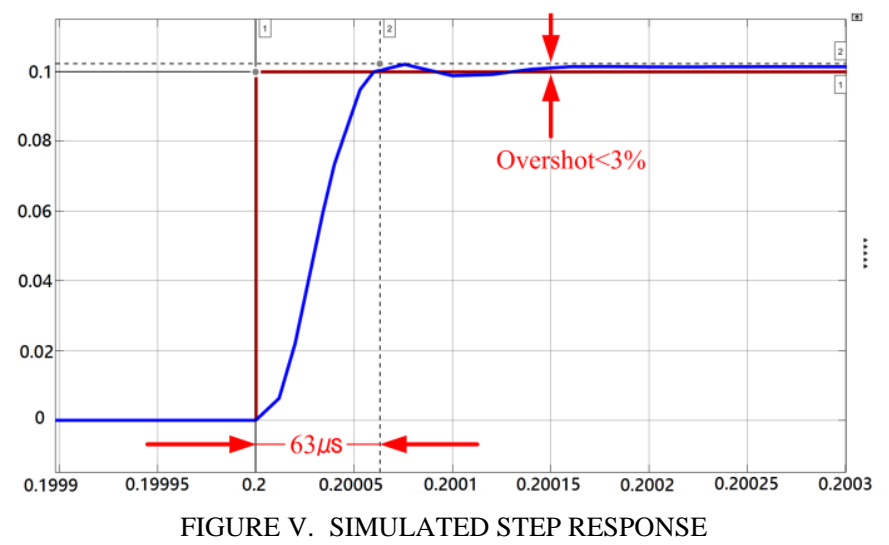

In the following section, the hardware implementation of the digital TPS fast corrector will be explained in detail using the PID controller designed as derived above.

\section{HARDWARE DESIGN}

The hardware architecture to realize the digital fast corrector power supply for the TPS ring is depicted in Figure VI. The digital power converter architecture consists of four building blocks : an NI embedded device with FPGA with digital IO ports, a single channel 18bits zero-latency successive approximation (SAR) ADC, a four channel DAC converters, and fast corrector power converter stage connected to a fast corrector magnet load.

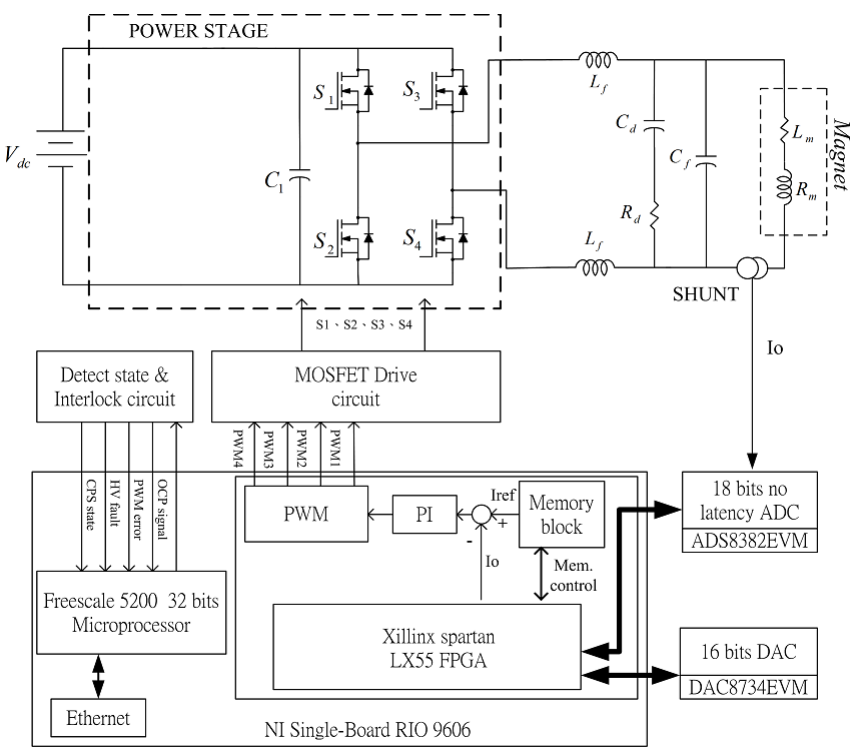

FIGURE VI. HARDWARE ARCHITECTURE OF DIGITAL FAST CORRECTOR POWER SUPPLY

The embedded controller bock is implemented with NI sbRIO-9606 single board computer equipped with Xilinx Spartan 6 FPGA and DIO (digital Input Output) ports, which performs the major tasks : 1) Input/Output data communication to accept synchronous trigger, current command profile and PID control parameters through Ethernet protocol. 2) Digital PID block and PWM signals generation. 3) ADC read-back control and 4) DACs output control for system parameters monitoring.

All the PID parameters and current command are sent to hardware module from PC through the Ethernet. The output current is measured and converted to voltage signal using 0.1 $\mathrm{Ohm}$ high precision shut resistor with $0.1 \%$ tolerance and $\pm 1 \mathrm{ppm} / \mathrm{K}$ temperature drift. The voltage signal is buffered and amplified with a gain of 10 and then amplified signal is sampled by zero-latency 18 Bits ADS8382 ADC with $50 \mathrm{KHz}$ sampling rate. The sampled current feedback data is retrieved back to the FPGA module to produce together with the current command the error signal to the digital PID block. The compensated command is calculated in PID and output to the PWM converter to generate the MOSFET switching signals for the H-bridge power stage. The four-channel DACs are used to observe the command input and tracking error for easy comparison purpose through external digital oscilloscope. The photo of the realized digital fast corrector power supply is shown in Figure VII. 


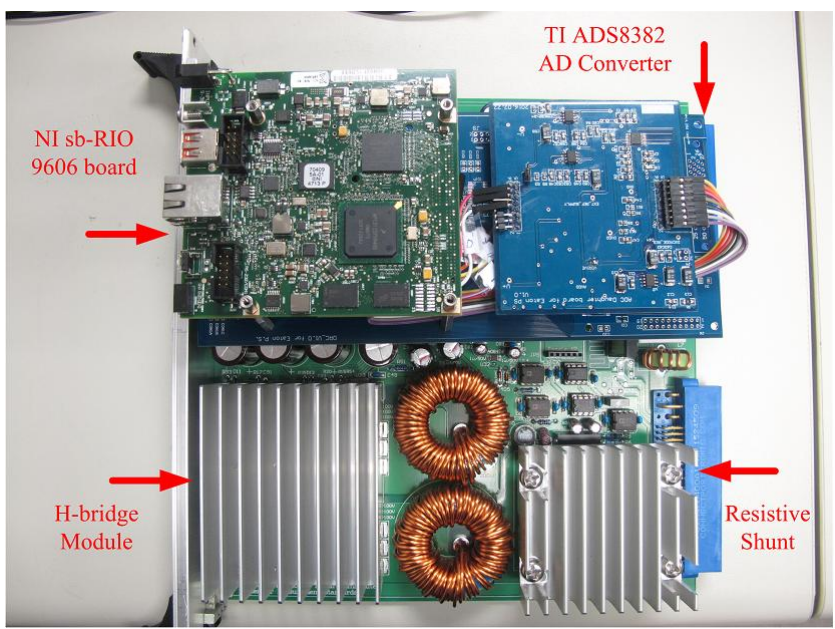

FIGURE VII. PHOTO OF THE RELIAZED PROTOTYPE

\section{EXPERIMENTAL RESULTS}

To validate the designed power supply, a prototype has been built and tested if the performances meet the design requirements. For external output current measurement, high precision, wide bandwidth DCCT unit (ITZ 600 ULTRASTAB, Danfysik) is employed. The output current together with the current command output from DAC are monitored by oscilloscope to observe the designed performance. Figure VIII shows the 0.1 A step response. The yellow line is the 0.1 A step command from DAC while the grey and blue one are the current output of the prototype digital and existing analog version of fast corrector power supply respectively. The rise time of the proposed digital prototype is about 68 us, which is slower than the 63 us simulated result but still falls well within the specified 100 us FOFB update interval. Furthermore, compared to 126 us rise time of the analog version, the rise time of the digital prototype is significantly improved.

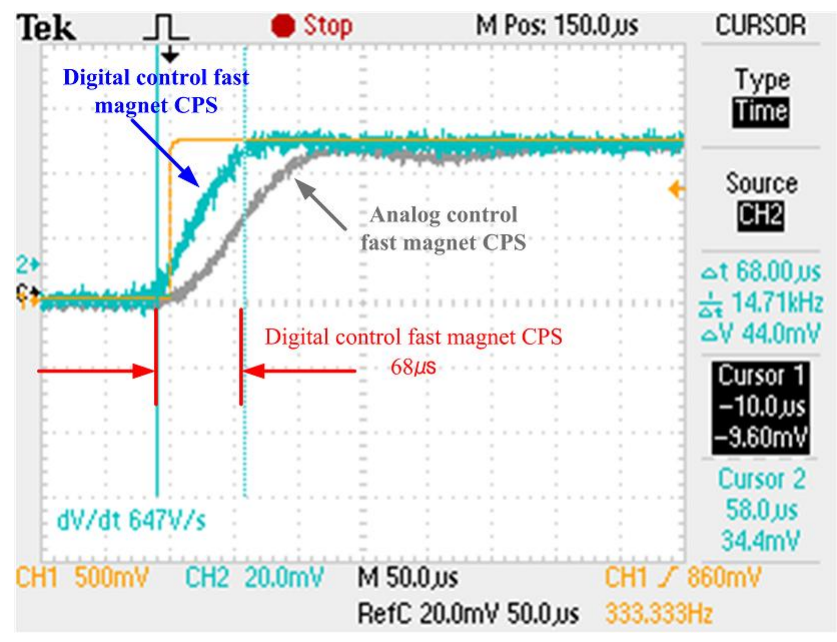

FIGURE VIII. MEASURED STEP RESPONSE.

Also the frequency response of the experimental prototype is measured and compared to the simulation as shown in Figure IX. The $-3 \mathrm{~dB}$ bandwidth of the prototype system is at 9.25 KHz. The measured phase margin is $160^{\circ}$ and the gain margin is $10 \mathrm{~dB}$, while the simulation results give $150^{\circ}$ and $4.98 \mathrm{~dB}$. From both the step and frequency responses, the performances of the proposed digital system is close to that of the mathematical model simulation and above all, meet the specifications as desired by the TPS FOFB system.

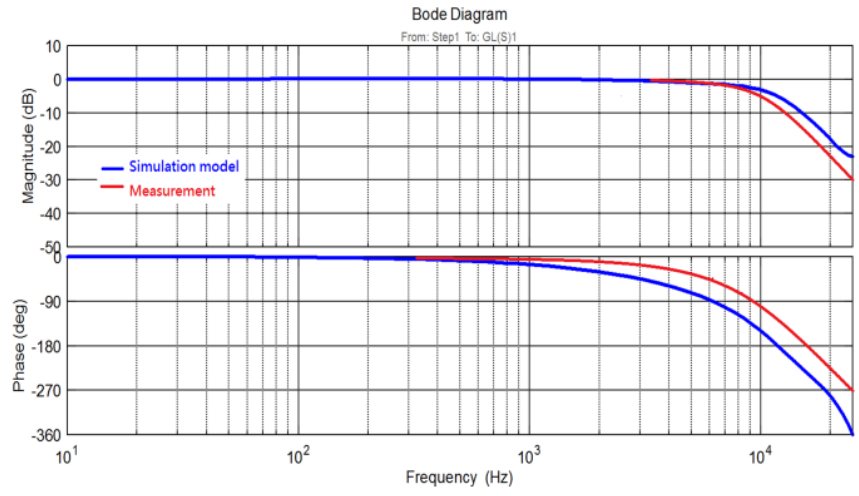

FIGURE IX. FREQUENCY RESPONSE OF THE SIMULATED MODEL AND MEASURED PROTOTYPE

\section{CONCLUSION}

This paper presents a preliminary digital power supply design for the TPS storage ring fast corrector based on off-theshelf embedded micro-controller board with FPGA and precision zero latency ADC. The proposed power supply control architecture is proved to be a compact current-mode controller with high bandwidth and fast transient. The experimental results also validate that the design satisfies the time-domain step response performance necessary for the FOFB system with $10 \mathrm{KHz}$ update rate. With the capability of adjusting the discrete PID gains more efficiently to adapt to various types of magnet loads, this implies that a more robust and reliable performance can be expected than the analog design one.

\section{REFERENCES}

[1] C. C. Kuo, et al. "Commissioning of the Taiwan Photon Source," Proceeding of IPAC 2015, Richmond, VA, USA, pp.1314-1318.

[2] O Singh, Y Tang, S Krinsky, "Orbit feedback at the NSLS," Nucl. Instrum. Meth. A., vol. 556, pp.267-279, 1998.

[3] P. C. Chiu, K. H. Hu, C.H. Huang,C. Y. Liao, K. T. Hsu, "Preliminary Beam Test for TPS Fast Orbit Feedback System," Proceeding of IPAC 2016, Busan , Korea, pp.2930-2932..

[4] K.B. Liu, Y.D. Li, B.S. Wang, K.T. Hsu, and J. C. Hsu, "TPS Corrector Magnet Power Converter," Proceeding of IPAC 2010, Kyoto , Japan, pp.3269-3271.

[5] R. Garcia-Gil, J. M. Espi, F. Voelker, E. J. Dede, J. Castello, "A novel four-quadrant power supply for low-energy correction magnets," Nucl. Instrum. Meth. A., vol. 510, pp.357-361, 2003.

[6] Jiang Zhao, et al. "Implementation of an FPGA controller for correction power supplies in heavy ion synchrotron," Nucl. Instrum. Meth. A., vol. 777, pp.167-171, 2015..

[7] M. Mohan, T. Undeland, W Robbins, Power Electronics. $3^{\text {rd }}$ Edition, John Wiley\& Sons, Inc. 2003 